\title{
1 Final Report for Utah State's SciDAC CEMM Contribution
}

This document represents a summary of work carried out at Utah State University in conjunction with the Center for Extended Magnetohyrodynamic Modeling (CEMM). The principal investigator, Dr. Eric Held, was aided in this work by two former graduate students, Drs. John James and Michael Addae-Kagyah, who completed their PhD's while being partially funded by CEMM monies. In addtion, Dr. Jeong-Young Ji, a postdoctoral researcher and Mukta Sharma, a graduate student were supported. This document collects the progress reports from all three years of this grant.

\section{Year 1 Progress Report for Utah State's Sci- DAC CEMM Contribution}

This is a progress report on Utah State's Year 1 contribution to the Center for Extended Magnetohydrodynamic Modeling SciDAC project. Work at Utah State is lead by Dr. Eric Held who is presently working with 3 graduate students and a post-doc funded by various grants including SciDAC.

\subsection{Implementation of $\pi_{\|}$For Ions in NIMROD}

As part of the SciDAC CEMM work, a unified form for the parallel ion stress, $\pi_{\|}$, in a magnetized plasma has recently been implemented in NIMROD. The analytic form, which was presented in the paper "Unified Form for Parallel Ion Viscous Stress in Magnetized Plasmas," E. D. Held, Physics of Plasmas, 10 (4708) 2003, involves coupled Volterra (integral) equations. Because the $\pi_{\|}$closure acted back on itself as a drive, its numerical implementation proved to be more difficult than the previous implementation of the general forms for the parallel electron and ion heat flows, $q_{\|}$. However, in the past year we have constructed a numerical scheme that calculates the parallel closures by using a fitting technique that rapidly approximates the thermodynamic drives (gradients in temperatures and ion flow). This technique is discussed in more detail below. With regards to the ion $\pi_{\|}$, this approach allows for an efficient, numerical solution to the coupled Volterra equations and hence a computationally efficient implementation in the NIMROD code.

\subsection{Efficient Numerical Implementation of $\pi_{\|}$and $q_{\|}$Clo- sures}

The fitting technique used to speed up the closure calculation is referred to as a Lomb periodogram. Essentially, while integrating parallel to the magnetic field, the temperatures and ion flow are stored at unequally spaced points. Using this data, the Lomb periodogram rapidly extracts the dominant wave numbers 
of the perturbations. These wave numbers are then used in a linear fit to find the sinusoidal coefficients that best represent the data. With this linear fit, the closures can then be calculated analytically. This technique has led to a speedup of the closure calculation by factors approaching 1000. The important result is that numerical simulations using NIMROD and the parallel closures which previously took hundreds of processors can now be preformed in minutes on a laptop. A demonstration of this was given at the 2005 Sherwood NIMROD meeting in Lake Tahoe.

\subsection{Hiring of Post-doc and Grad Student}

In another important development regarding CEMM SciDAC funds, a post-doc, Jeong-Young Ji, and a graduate student, Mukta Sharma, have been hired. Ms. Sharma has begun her $\mathrm{PhD}$ research as a research assistant in the USU fusion group as of May 1st. Upon finishing his thesis defense, Dr. Ji will also officially join the group starting in the middle of June. These additions will greatly assist progress on the parallel closure work that is an integral part of the CEMM project. We look forward to updating you on further progress associated with the CEMM project. Thank you.

\section{Year 2 Report for Utah State's SciDAC CEMM Contribution}

This is a progress report on Utah State's Year 2 contribution to the Center for Extended Magnetohydrodynamic Modeling SciDAC project. Work at Utah

State is lead by Dr. Eric Held who is presently working with 3 graduate students and a post-doc funded by various grants including SciDAC.

\subsection{Physics tests using integral $q_{\|}$and $\pi_{\|}$in NIMROD}

As part of the SciDAC CEMM work, the numerical implementation of unified forms for the parallel heat flows, $q_{\|}$, and ion stress, $\pi_{\|}$, in a magnetized plasma are being tested in NIMROD on basic physics problems of interest. The first problem involves heat flow for overlapping magnetic islands in slab geometry. The signature of temperature perturbations along magnetic field lines in this geometry is nonlinear and complicated. The successful fitting technique implemented as part of first year SciDAC CEMM work is employed to accelerate calculation of $q_{\|}$. The importance of nonlocal effects will be emphasized in an near term paper where comparison will be made to the predictions of the local (Braginskii) parallel heat flow closure. The second physics application is the study of parallel sound wave damping due to the integral $\pi_{\|}$closure. A careful study of the modification to the damping as one proceeds from the collisional to the collisionless regime will also result in a near term paper. Both of these problems serve as preparation for the more difficult neoclassical tearing mode (NTM) studies which will include both $q_{\|}$and $\pi_{\|}$effects. These studies will be carried out in the upcoming year of SciDAC CEMM work at Utah State.

\subsection{Derivation of neoclassical parallel closures}

Previous analytic work involving toroidal geometry (neoclassical) effects emphasized the $q_{\|}$closure and temperature gradient drive. Presently, Mukta Sharma (graduate student) is working on a derivation that will represent a comprehensive parallel closure scheme for the electron and ion $q_{\|}$'s and $\pi_{\|}$'s that include particle trapping, free-streaming and collisional effects. With regards to collisional effects, an improved moment approach to handling the linearized collision operator (worked performed by postdoc Jeong-Young Ji in conjunction with the PSI-Center) will be used in this derivation. This analytic work will result in improved closures in NIMROD for the NTM simulations mentioned above. 


\subsection{Conclusion}

Dr. Ji and Mrs. Sharma will be working primarily on the CEMM project in the next year, hence it is anticipated that progress will be swift and significant. Thanks for your consideration and continued financial support.

\section{Year 3 Progress Report for Utah State's Sci- DAC CEMM Contribution}

This is a final progress report on Utah State's contribution to the Center for Extended Magnetohydrodynamic Modeling SciDAC project. Work at Utah State is lead by Dr. Eric Held who is presently working with a graduate student, Mukta Sharma, and a postdoc, Dr. Jeong-Young Ji, funded by various grants including CEMM.

\subsection{Parallel scaling of closures in NIMROD}

Previous implementations of the parallel closures in NIMROD had all processors performing both the fluid advance and the closure calculation. This tended to limit the scaling of the overall calculation to that of the fluid advance. A new implementation uses the MPI split command to separate the parallelism of the closure calculation from that of the fluid advance allowing for large groups (1000's) of processors to independently compute the closures.

As part of NERSC's ERCAP process, scaling runs were performed using NIMROD in conjunction with the parallel, integral heat flow closure. In the interest of understanding electron heat confinement in the presence of stochastic magnetic fields for SSPX, temperature was evolved to steady state keeping the magnetic field fixed in time. The core temperature results from balancing parallel and perpendicular transport against resistive heating. The parallel heat flow closure calculation entails solving the steady state electron drift kinetic equation approximately 1 million times throughout the computational domain before the temperature is advanced. Figure 1 shows good scaling up to 2048 processors with an efficiency of about $75 \%$. Up to 1024 processors, efficiency is nearly perfect at $90 \%$. One possible reason for the fall off beyond 1000 processors is the fact that processors added beyond the 1024 mark were solely used for the closure calculation. Although this part of the problem scales perfectly, the total time (summed over processors) spent waiting for the updated temperature from the fluid processors increases. Improving the scaling beyond 2000 processors most likely requires increasing the fluid processors by the same factor as the closure processors and being in regime where the fluid part scales perfectly. Future improvements of scaling with the integral parallel closures are planned for the next round of CEMM activities including performing scaling studies on Franklin and possibly Jaguar.

\subsection{Derivation of neoclassical parallel closures}

Previous analytic work involving toroidal geometry (neoclassical) effects emphasized the parallel heat flow closure, $q_{\|}$, and temperature gradient drive. Presently, Mukta Sharma is working on a derivation that will represent a comprehensive parallel closure scheme for the electron and ion parallel heat flows, $q_{\|}$'s, and parallel stresses, $\pi_{\|}$'s, including particle trapping, free-streaming and collisional effects. With regards to collisional effects, an improved moment approach to handling the linearized collision operator (worked performed by Drs. $\mathrm{Ji}$ and Held in conjunction with the PSI-Center) is being used in this derivation. In addition, we are not bounce-averaging the kinetic equation as part of the solution process. Rather, the portion of the free-streaming operator that brings in the effects of toroidal geometry are treated using a moment approach in this theory. Preliminary work involving tent wells for the magnitude of the magnetic field is underway. This work will indicate whether a moment approach for the neoclassical physics is appropriate. The end result of this analytic work 


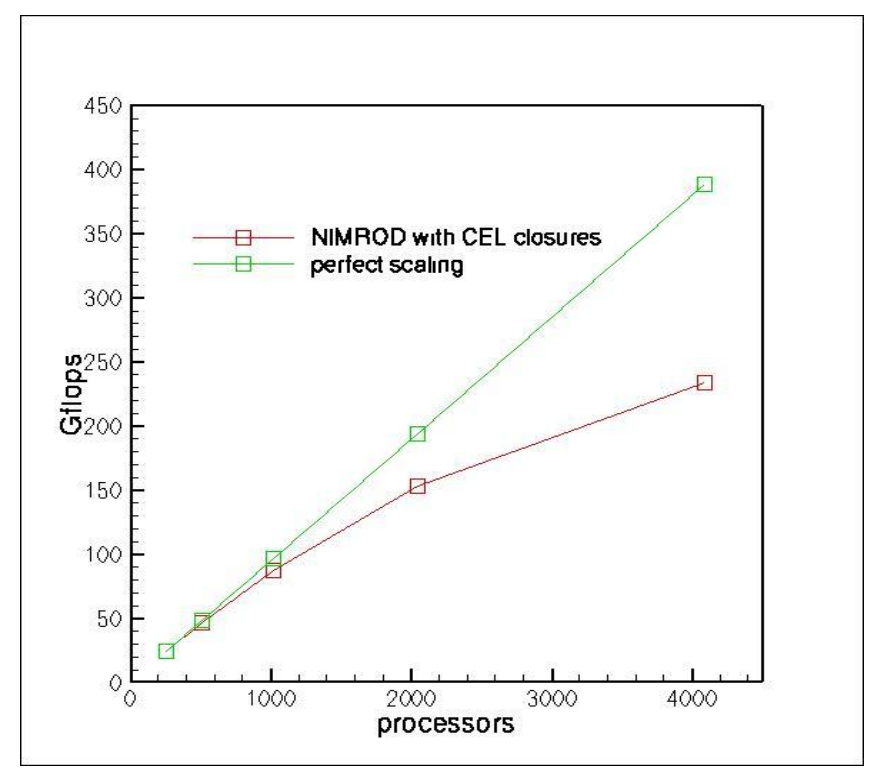

Figure 1: Figure 1: Scaling of the parallel heat flow closure calculation showing performance in Gflops as processors are added. Note the efficient scaling to thousands of processors.

will be a comprehensive closure model for neoclassical tearing mode (NTM) simulations, as well as simulations of other instabilities where toroidal geometry is important, using the NIMROD code.

\subsection{Conclusion}

As we move into the next round of funding for CEMM, we are nearing completion of an efficient, massively parallel, comprehensive closure model. This model will be essential for producing novel simulation results of NTMs and other instabilities in tokamaks. Thanks for your consideration and continued financial support. 